\title{
Congress Calendar
}

\section{Respirition}

\begin{tabular}{l}
\hline 1.11.-3.11.2007 \\
Kanagawa \\
Japan \\
\hline 4.11.-7.11.2007 \\
Orlando, Fla. \\
USA \\
\hline 6.11.-11.11.2007 \\
Fethlye \\
Turkey \\
\hline 1.12.-4.12.2007 \\
Orlando, Fla. \\
USA \\
\hline 2.12.-6.12.2007 \\
Bangkok \\
Thailand \\
\hline 4.12.-7.12.2007 \\
Marseille \\
France \\
\hline 5.12.-7.12.2007 \\
London \\
UK \\
\hline 5.12.-7.12.2007 \\
Cairo \\
Egypt \\
\hline 29.3.-31.3.2008 \\
Nice \\
France \\
\hline 9.4.-12.4.2008 \\
Lübeck \\
Germany \\
\hline 17.4.-18.4.2008 \\
Fribourg \\
Switzerland \\
\hline
\end{tabular}

19.4.-22.4.2008

Barcelona

Spain

26.4.-29.4.2008

Dubai

United Arab Emirates

16.5.-21.5.2008

Toronto

Canada
Japanese Society of Allergology - 57th Annual Meeting

American Heart Association

Turkish Society for Respiratory Investigators

29th Annual Congress

American Association for Respiratory Care

53rd International Respiratory Congress

World Allergy Congress

Thorascopy \& Pleural Techniques

British Thoracic Society Winter Meeting

7th Annual International Conference of The Egyptian Society of Bronchology

8th International Congress on Pediatric Pulmonology

49. Jahreskongress der Deutschen Gesellschaft für Pneumologie und Beatmungsmedizin

Gemeinsame Jahresversammlung

Schweizerische Gesellschaft für Pneumologie

Schweizerische Gesellschaft für Pädiatrische Pneumologie Schweizerische Gesellschaft für Allergologie und Immunologie Schweizerische Gesellschaft für Thoraxchirurgie

18th European Congress of Clinical Microbiology and Infectious Diseases

World Asthma \& COPD Forum

ATS 2008 - American Thoracic Society

Future ATS Meetings:

15.5.-20.5.2009, San Diego, Calif., USA

14.5.-19.5.2010, New Orleans, La., USA

13.5.-18.5.2011, Denver, Colo., USA
Information: http://www.jsaweb.jp/english/index.html

Information: http://scientificsessions.americanheart. org/portal/scientificsessions/ss/

Information: E-Mail info@solunum.org.tr, Internet www.solunum.org.tr

Information: www.aarc.org

Information: www.wac2007.com

Information: school@ersnet.org

Information: http://www.brit-thoracic.org.uk/

bts_winter_meetings.html

Information: E-Mail ahatem@alfapneumocare.com Internet www.essbronchology.com

Information: www.cipp-meeting.com

Information: http://www.pneumologie.de/ Jahreskongresse-DGP_24.html

Information: http://www.akm.ch/sgp-sgai2008/

Information: E-Mail info@akm.ch,

Internet: www.escmid.org/eccmid2008

Information: www.wipocis.org

Information: www.thoracic.org 
11.6.-13.6.2008

Birmingham

UK

12.6.-14.6.2008

Ljubljana

Slovenia

19.6.-22.6.2008

Athens

Greece

18.9.-20.9.2008

Wien

Austria

4.10.-8.10.2008

Berlin

Germany

25.10.-30.10.2008

Philadelphia, Pa.

USA

5.11.-8.11.2008

Monte Carlo

Monaco
The 6th International Meeting on COPD

11 th Central European Lung Cancer Conference

9th WASOG Meeting \& 11 th BAL International Conference

World Association of Sarcoidosis and Other Granulomatous Diseases

(WASOG)

Bronchoalveolar Lavage (BAL)

Endorsed by the European Respiratory Society

Jahrestagung der Österreichischen Gesellschaft für Pneumologie (ÖGP)

ERS 18th Annual Congress

Future ERS Annual Congress

12.9.-16.9.2009, Vienna, Austria

CHEST 2008

The American College of Chest Physicians

Future CHEST Assemblies:

CHEST 2009 - 31.10.-5.11.2009, San Diego, Calif., USA

XIX World Congress of Asthma
Information: E-Mail info@copdconferences.org,

Internet: www.copdconferences.org

Information: http://en.ce-lung2008.org

Information: www.wasogbal2008.com

Information: http://www.ogp.at/

Information: ERS Headquarters, 4, Avenue Ste. Luce, CH-1003 Lausanne (Switzerland),

Tel. +4121213 0101, Fax +41212130100,

E-Mail info@ersnet.org,www.ersnet.org

Information: ACCP Member Services,

3300 Dundee Road, Northbrook, IL 60062 (USA),

Tel. +1 847498 1400, Fax +1 847498 5460,

E-Mail registrationschestnet.org, www.chestnet.org

Information: www.aim-internationalgroup.com/ 2008/wca 